\title{
MARTINGALES FOR QUASISYMMETRIC SYSTEMS AND COMPLEX MANIFOLD STRUCTURES
}

\author{
Yunchun Hu, Yunping Jiang and Zhe Wang \\ CUNY Graduate Center, Department of Mathematics \\ New York, NY 10016, U.S.A.; yhu@ccny.cuny.edu \\ CUNY Queens College and Graduate Center, Department of Mathematics \\ Flushing, NY 11367, U.S.A.; Yunping.Jiang@qc.cuny.edu \\ Bronx Community College, Department of Mathematics and Computer Science \\ New York, NY 10453, U.S.A.; wangzhecuny@gmail.com
}

\begin{abstract}
We construct an infinite martingale sequence on the dual symbolic space from a uniformly quasisymmetric circle endomorphism preserving the Lebesgue measure. This infinite martingale sequence is uniformly bounded. Thus from the martingale convergence theorem, there is a limiting martingale which is the unique $L^{1}$ limit of this uniformly bounded infinite martingale sequence. Moreover, we prove that the classical Hilbert transform gives an almost complex structure on the space of all uniformly quasisymmetric circle endomorphisms preserving the Lebesgue measure. Furthermore, we discuss the complex manifold structure which is the integration of the almost complex structure. We further discuss the comparison between the global Kobayashi's metric and the global Teichmüller metric on the fiber of the forgetful map at the basepoint. We prove that these two metrics are not equivalent.
\end{abstract}

\section{Introduction}

Tossing a coin is an old game in the gambling business. For a fair coin, the head and the tail of the coin have equal chances to occur. From the modern mathematical language of Kolmogorov, the probability of the head or the tail is $1 / 2$ when one tosses a fair coin. If one tosses a fair coin $n$-times, there are $2^{n}$ outcome events. Each event has the probability $1 / 2^{n}$. The relative probability from the $(n-1)^{\text {th }}$ tossing to the $n^{\text {th }}$ tossing is still $1 / 2$ because they are independent events. Thus if one let $X_{n}=1 / 2$ denote the relative probability of the $n^{\text {th }}$ tossing by given the result of the $(n-1)^{\text {th }}$ tossing. It is a random variable. The sequence of random variables $\left\{X_{n}\right\}_{n=1}^{\infty}$ thus forms an infinite martingale sequence. The limiting martingale exists and is a random variable $X=1 / 2$ defined on the space of all infinite sequences of head's and tail's. Therefore, from the long term predication point of view, a fair coin is predicable.

doi:10.5186/aasfm.2013.3812

2010 Mathematics Subject Classification: Primary 37E10, 37F15, 37F30, 30F60.

Key words: Martingale, quasisymmetric homeomorphism, uniformly quasisymmetric circle endomorphism, Hilbert transform.

The research of the second and the third authors were supported by PSC-CUNY grants. The second author was partially supported by the collaboration grant (\#199837) from the Simons Foundation, the CUNY collaborative incentive research grant (\#1861), a grant (\#11171121) from the NSF of China and a collaboration grant from Academy of Mathematics and Systems Science and the Morningside Center of Mathematics at the Chinese Academy of Sciences. And the third author was also supported by CUNY community college collaborative incentive research grant (\#24210). 
If one tosses an infinite sequence of unfair coins, the story will be completely different. The long term predication could fail. The main purpose of this paper is to make sure that the long term predication is still valid if we propose a quasisymmetric condition (see (1)) and an invariant condition (see (6)). That is, under the quasisymmetric condition and the invariant condition, we have a uniformly bounded infinite martingale sequence whose limit exists and is a bounded random variable. More importantly, the quasisymmetric property enables us to construct a Teichmüller structure and an almost complex structure over the space of all these martingales using the universal Teichmüller space. Therefore, the long term prediction for the infinite sequences of unfair coins which are considered in this paper not only are predicable but also together have a well defined complex manifold structure. We further study some properties about the almost complex manifold structure and the complex manifold structure. In particular, we prove that the complex manifold structure is the integration of the almost complex structure. The comparison between the global Kobayashi's metric and the global Teichmüller metric on the fiber of the forgetful map at the basepoint is also studied. We prove that these two metrics are not equivalent. This is an opposite result to a result obtained by Gardiner and Lakic in [9] (see Theorem 12). Their result says that the infinitesimal Kobayashi's density and the infinitesimal Teichmüller density on the fiber of the forgetful map at the basepoint are equivalent.

The paper is organized as follows. In $\S 2$, we study uniformly quasisymmetric circle endomorphisms fixing 1 . In $\S 3$, we give the symbolic coding for all uniformly quasisymmetric circle endomorphisms with a fixed degree. In $\S 4$, we study the bounded geometry and bounded nearby geometry of a sequence of nested Markov partition. In $\S 5$, we give a brief review of the theory of martingales in the probability theory. In $\S 6$, we construct the uniformly bounded infinite martingale sequence for every uniformly quasisymmetric circle endomorphism preserving the Lebesgue measure and prove that the limiting martingale exists and is a $L^{1}$ limit of the uniformly bounded infinite martingale sequence. In $\S 7$, we prove that the classical Hilbert transform gives an almost complex structure on the space of all uniformly quasisymmetric circle endomorphisms fixing 1 and preserving the Lebesgue measure. In $\S 8$, we construct the complex manifold structure on the space of all uniformly quasisymmetric circle endomorphisms fixing 1 and preserving the Lebesgue measure through the construction of the complex manifold structure on the space of all uniformly quasisymmetric circle endomorphisms fixing 1 . We prove that this complex manifold structure is the same as the complex manifold structure of the Teichmüller space of Riemann surfaces with the punctured disk as the basepoint. We also show that this complex manifold structure is the integration of the almost complex manifold structure. There is the forgetful map from the Teichmüller space of Riemann surfaces with the punctured disk as the basepoint to the universal Teichmüller space. The fiber of the basepoint is a one dimensional complex manifold. Gardiner and Lakic proved that on this fiber, the infinitesimal Kobayashi's density and the infinitesimal Teichmüller density at the basepoint are equivalent. We give a proof of this result in $\S 9$ (see Theorem 12). More importantly, in $\S 9$, we prove that globally, this is not true. More precisely, in $\S 9$, we study the comparison between the global Kobayashi's metric and the global Teichmüller metric on the fiber of the forgetful map at the basepoint. We prove that these two metrics are not equivalent (see Theorem 13). 
Acknowledgements. We would like to express our thanks to Fred Gardiner for many inspiration conversations and emails. We would also like to thank Tao Chen, Xiaojun Huang, and Sudeb Mitra for their patience to listen our computations for many hours. We would also like to thank the anonymous referee for many comments and suggestions which we have included into this new version.

\section{Uniformly quasisymmetric circle endomorphisms}

Let $T=\{z \in \mathbf{C}|| z \mid=1\}$ be the unit circle in the complex plane C. Suppose

$$
f: T \rightarrow T
$$

is an orientation-preserving covering map of degree $d \geq 2$. We call it a circle endomorphism. Suppose

$$
h: T \rightarrow T
$$

is an orientation-preserving homeomorphism. We call it in this paper a circle homeomorphism.

For a circle endomorphism $f$, it has a fixed point. By conjugating a rotation of the circle, we will always assume that 1 is a fixed point of $f$, that is, $f(1)=1$.

The universal cover of $T$ is the real line $\mathbf{R}$ with a covering map

$$
\pi(x)=e^{2 \pi i x}: \mathbf{R} \rightarrow T .
$$

Then every circle endomorphism $f$ can be lifted to an orientation-preserving homeomorphism

$$
F: \mathbf{R} \rightarrow \mathbf{R}, \quad F(x+1)=F(x)+d, \quad \forall x \in \mathbf{R} .
$$

We will assume that $F(0)=0$. Then there is a one-to-one correspondence between $f$ and $F$. Therefore, we also call such an $F$ a circle endomorphism.

Every orientation-preserving circle homeomorphism $h$ can be lifted to an orientationpreserving homeomorphism

$$
H: \mathbf{R} \rightarrow \mathbf{R}, \quad H(x+1)=H(x)+1, \quad \forall x \in \mathbf{R} .
$$

We will assume throughout this paper that $0 \leq H(0)<1$. Then there is a one-toone correspondence between $h$ and $H$. Therefore, we also call such an $H$ a circle homeomorphism.

Definition 1. A circle homeomorphism $h$ is called quasisymmetric if there is a constant $M \geq 1$ such that

$$
M^{-1} \leq \frac{|H(x+t)-H(x)|}{|H(x)-H(x-t)|} \leq M, \quad \forall x \in \mathbf{R}, \forall t>0 .
$$

Definition 2. A circle endomorphism $f$ is called uniformly quasisymmetric if there is a constant $M>0$ such that

$$
M^{-1} \leq \frac{\left|F^{-n}(x+t)-F^{-n}(x)\right|}{\left|F^{-n}(x)-F^{-n}(x-t)\right|} \leq M
$$

for all $x \in \mathbf{R}$ and $t>0$ and any $n>0$.

The following example of a uniformly quasisymmetric circle endomorphisms can be found in $[13,19]$. 
A circle endomorphism $f$ is $C^{1}$ if the first derivative $F^{\prime}$ exists and is continuous. And, furthermore, it is called $C^{1+\alpha}$ for some $0<\alpha \leq 1$ if $F^{\prime}$ is $\alpha$-Hölder continuous, that is,

$$
\sup _{x \neq y \in \mathbf{R}} \frac{\left|F^{\prime}(x)-F^{\prime}(y)\right|}{|x-y|^{\alpha}}=\sup _{x \neq y \in[0,1]} \frac{\left|F^{\prime}(x)-F^{\prime}(y)\right|}{|x-y|^{\alpha}}<\infty .
$$

A $C^{1}$ circle endomorphism $f$ is called expanding if there are constants $C>0$ and $\lambda>1$ such that

$$
\left(F^{n}\right)^{\prime}(x) \geq C \lambda^{n}, \quad n=1,2, \cdots .
$$

Example 1. A $C^{1+\alpha}$, for some $0<\alpha \leq 1$, circle expanding endomorphism $f$ is uniformly quasisymmetric. $d$. Let

Consider the map $q(z)=z^{d}$ for $d>1$. Then it is a circle endomorphism of degree

$$
f=h \circ q \circ h^{-1}
$$

where $h$ is a circle homeomorphism.

Theorem 1. The circle endomorphism $f$ is uniformly quasisymmetric if and only if $h$ is a quasisymmetric homeomorphism.

Refer to $[17,19]$ (or refer to $[12,13]$ ) for this theorem. Thus, we consider two spaces

$\mathcal{F}=\{f \mid f$ is a uniformly quasisymmetric circle endomorphism with $f(1)=1\}$ and

$$
\mathcal{H}=\{h \mid h \text { is a quasisymmetric circle homeomorphism with } h(1)=1\} .
$$

The map

$$
\beta: \mathcal{H} \rightarrow \mathcal{F} ; \quad \beta(h)=h \circ q \circ h^{-1}
$$

is a bijective map (see [17]).

\section{Circle endomorphisms and their symbolic representation}

Suppose $f$ is a circle endomorphism in $\mathcal{F}$. Consider the preimage $f^{-1}(1)$. Then $f^{-1}(1)$ cuts $T$ into $d$ closed intervals $J_{0}, J_{1}, \cdots, J_{d-1}$, ordered by the counter-clockwise order of $T$. Suppose $J_{0}$ has an endpoint 1 . Then $J_{d-1}$ also has an endpoint 1 . Let

$$
\varpi_{0}=\left\{J_{0}, J_{1}, \cdots, J_{d-1}\right\} .
$$

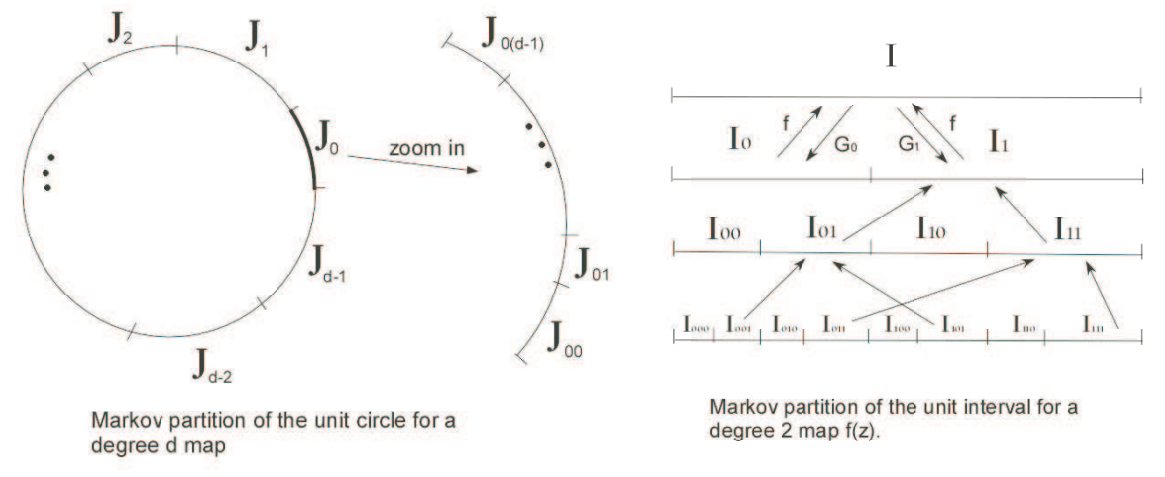

Figure 1. 
Then it is a Markov partition, that is,

i. $T=\bigcup_{k=0}^{d-1} J_{k}$,

ii. the restriction of $f$ to the interior of $J_{i}$ is injective for every $0 \leq i \leq d-1$,

iii. $f\left(J_{i}\right)=T$ for every $0 \leq i \leq d-1$.

Let $I_{0}, I_{1}, \cdots, I_{d-1}$ be the lifts of $J_{0}, J_{1}, \cdots, J_{d-1}$ in $[0,1]$. Then we have that

i) $[0,1]=\bigcup_{k=0}^{d-1} I_{k}$,

ii) $F\left(I_{i}\right)=[i, i+1]$ for every $0 \leq i \leq d-1$.

Let

$$
\eta_{0}=\left\{I_{0}, I_{1}, \cdots, I_{d-1}\right\} .
$$

Then it is a partition of $[0,1]$.

Consider the pull-back partition $\varpi_{n}=f^{-n} \varpi_{0}$ of $\varpi_{0}$ by $f^{n}$. It contains $d^{n+1}$ intervals and is also a Markov partition of $T$. Intervals $J$ in $\varpi_{n}$ can be labeled as follows. Let $w_{n}=i_{0} i_{1} \cdots i_{n-1}$ be a word of length $n$ of $0^{\prime} s, 1^{\prime} s, \cdots$, and $(d-1)^{\prime} s$. Then $J_{w_{n}} \in \varpi_{n}$ if $f^{k}\left(J_{w_{n}}\right) \subset J_{i_{k}}$ for $0 \leq k \leq n-1$. Then

$$
\varpi_{n}=\left\{J_{w_{n}} \mid w_{n}=i_{0} i_{1} \cdots i_{n-1}, i_{k} \in\{0,1, \cdots, d-1\}, k=0,1, \cdots, d-1\right\} .
$$

Let $\eta_{n}$ be the corresponding lift partition of $\varpi_{n}$ in $[0,1]$ with the same labelings. Then

$$
\eta_{n}=\left\{I_{w_{n}} \mid w_{n}=i_{0} i_{1} \cdots i_{n-1}, i_{k} \in\{0,1, \cdots, d-1\}, k=0,1, \cdots, d-1\right\} .
$$

Add a digit at the end means subinterval and add a digit in the front means preimage.

Consider the space

$$
\begin{aligned}
\Sigma & =\prod_{n=0}^{\infty}\{0,1, \cdots, d-1\} \\
& =\left\{w=i_{0} i_{1} \cdots i_{k} \cdots i_{n-1} \cdots \mid i_{k} \in\{0,1, \cdots, d-1\}, k=0,1, \cdots\right\}
\end{aligned}
$$

with the product topology. It is a compact topological space. A cylinder for a fixed word $w_{n}=i_{0} i_{1} \cdots i_{n-1}$ of length $n$ is

$$
\left[w_{n}\right]=\left\{w^{\prime}=i_{0} i_{1} \cdots i_{n-1} i_{n}^{\prime} i_{n+1}^{\prime} \cdots \mid i_{n+k}^{\prime} \in\{0,1, \cdots, d-1\}, k=0,1, \cdots\right\} .
$$

All left cylinders form a topological basis of $\Sigma$. We call it the left topology. The space $\Sigma$ with this left topology is called the symbolic space.

For any $w=i_{0} i_{1} \cdots i_{n-1} i_{n} \cdots$, let

$$
\sigma(w)=i_{1} \cdots i_{n-1} i_{n} \cdots
$$

be the shift map. Then $(\Sigma, \sigma)$ is called a symbolic dynamical system.

For a point $w=i_{0} \cdots i_{n-1} i_{n} \cdots \in \Sigma$, let $w_{n}=i_{0} \cdots i_{n-1}$. Then

$$
\cdots \subset J_{w_{n}} \subset J_{w_{n-1}} \subset \cdots J_{w_{1}} \subset T \text {. }
$$

Since each $J_{w_{n}}$ is compact,

$$
J_{w}=\bigcap_{n=1}^{\infty} J_{w_{n}} \neq \emptyset .
$$

If every $J_{w}=\left\{x_{w}\right\}$ contains only one point, then we define the projection $\pi_{f}$ from $\Sigma$ onto $T$ as

$$
\pi_{f}(w)=x_{w}
$$


The projection $\pi_{f}$ is $1-1$ except for a countable set

$$
B=\left\{w=i_{0} i_{1} \cdots i_{n-1} 1000 \cdots, i_{0} i_{1} \cdots i_{n-1} 0(d-1)(d-1)(d-1) \cdots\right\} .
$$

From our construction, one can check that

$$
\pi_{f} \circ \sigma(w)=f \circ \pi_{f}(w), \quad w \in \Sigma .
$$

This situation, we say that $f$ is semi-conjugate to $\sigma$ by the semi-conjugacy $\pi_{f}$.

For any interval $I=[a, b]$ in $[0,1]$, we use $|I|=b-a$ to mean its Lebesgue length. Let

$$
\iota_{n, f}=\max _{w_{n}}\left|I_{w_{n}}\right|,
$$

where $w_{n}$ runs over all words of $\{0,1, \cdots, d-1\}$ of length $n$.

Two circle endomorphisms $f$ and $g$ are topologically conjugate if there is an orientation-preserving circle homeomorphism $h$ of $T$ such that

$$
f \circ h=h \circ g \text {. }
$$

The following result was first proved by Shub for $C^{2}$ expanding circle endomorphisms 1960 's by using the contracting mapping theorem.

Theorem 2. Let $f$ and $g$ be two circle endomorphisms such that both $\iota_{n, f}$ and $\iota_{n, g}$ tend to zero as $n \rightarrow \infty$. Then $f$ and $g$ are topologically conjugate if and only if their topological degrees are the same.

Refer to $[19,17]$ for a proof.

From the bounded nearby geometry property for any $f \in \mathcal{F}$ which we will discuss in the next section, we have that $\iota_{n, f}$ tends to zero as $n \rightarrow \infty$ for any $f \in \mathcal{F}$. And from Theorem 1, we have that

Theorem 3. For any $f, g \in \mathcal{F}, f$ and $g$ are topologically conjugate. And the conjugacy $h$ (that is, $h \circ f=g \circ h$ ) is quasisymmetric. Furthermore, $f$ is always semi-conjugate to $\sigma$ by the semi-conjugacy $\pi_{f}$.

From the above theorem the symbolic dynamical system $(\Sigma, \sigma)$ is the topological representation for all maps in $\mathcal{F}$.

\section{Bounded nearby geometry}

In this section, we show that the uniformly quasisymmetric condition is equivalent to the bounded nearby geometry defined in [11, 12, 13].

Definition 3. The sequence $\left\{\varpi_{n}\right\}_{n=0}^{\infty}$ of nested partitions of $T$ is said to have bounded geometry if there is a constant $C>0$ such that

$$
\frac{\left|J_{\sigma\left(w_{n}\right)}\right|}{\left|J_{w_{n}}\right|} \leq C, \quad \forall J \in \varpi_{n} ; \quad \forall n \geq 0 .
$$

Definition 4. The sequence $\left\{\varpi_{n}\right\}_{n=0}^{\infty}$ of nested partitions of $T$ is said to have bounded nearby geometry if there is a constant $C>0$ such that

$$
\frac{|J|}{\left|J^{\prime}\right|} \geq C, \quad \forall J, J^{\prime} \in \varpi_{n} \text { with a common endpoint; } \quad \forall n \geq 0 .
$$

Theorem 4. Suppose $f$ is a circle endomorphisms. Then $f$ is uniformly quasisymmetric if and only if the sequence $\left\{\varpi_{n}\right\}_{n=0}^{\infty}$ of nested partitions of $T$ has the bounded nearby geometry. 
Proof. We first prove the "only if" part. Let $F$ with $F(0)=0$ be the lift of $f$. Define

$$
G_{k}(x)=F^{-1}(x+k):[0,1] \rightarrow[0,1], \quad \text { for } \quad k=0,1, \cdots, n-1 .
$$

For any word $w_{n}=i_{0} i_{1} \cdots i_{n-1}$, define

$$
G_{w_{n}}=G_{i_{0}} \circ G_{i_{1}} \circ \cdots \circ G_{i_{n-1}} .
$$

Then

$$
I_{w_{n}}=G_{w_{n}}([0,1])=F^{-n}([m, m+1]),
$$

where $m=i_{n-1}+i_{n-2} d+\cdots+i_{0} d^{n-1}$. Suppose $I_{w_{n}}^{\prime}$ is an interval in $\eta_{n}$ having a common endpoint with $I_{w_{n}}$ modulo 1 . Then

$$
I_{w_{n}}^{\prime}=F^{-n}([m+1, m+2]) \quad \text { or } \quad F^{-n}([m, m+1]) .
$$

Thus

$$
C^{-1} \leq \frac{\left|I_{w_{n}}\right|}{\left|I_{w_{n}}^{\prime}\right|} \leq C
$$

where $C>0$ is the constant in Definition 2. Since $I_{w_{n}}$ and $I_{w_{n}}^{\prime}$ are just lifts of $J$ and $J^{\prime}$, we have

$$
C^{-1} \leq \frac{|J|}{\left|J^{\prime}\right|} \leq C
$$

for any intervals $J, J^{\prime} \in \varpi_{n}$ with a common endpoint and $n=0,1, \cdots$.

The "if" part can follows the exact argument in [12, Theorem B, pp.645-646] (also, refer to [13, pp.90-91]). We give a outline of the proof here. Consider $f=$ $h \circ q \circ h^{-1}$. Recall that $q(z)=z^{d}$ where $d>1$ is the degree of $f$. Then from Theorem $1, f$ is uniformly quasisymmetric if and only if $h$ is quasisymmetric. Let $\varpi_{n, q}$ be the $n^{\text {th }}$-partition for $q$ and $\varpi_{n}$ be the $n^{\text {th }}$-partition for $f$. For any intervals $J, J^{\prime} \in \varpi_{n, q}$ with a common endpoint and any $n=0,1, \cdots$, we have $|J| /\left|J^{\prime}\right|=1$ and $h(J), h\left(J^{\prime}\right) \in \varpi_{n}$ with a common endpoint. The bounded nearby geometry says that we have a constant $C>0$, independent of $J, J^{\prime}$, and $n$, such that

$$
C^{-1} \leq \frac{|h(J)|}{\left|h\left(J^{\prime}\right)\right|} \leq C
$$

This says that $h$ is quasisymmetric at the common endpoint of $J$ and $J^{\prime}$ with a fixed quasisymmetric constant $C$. But these common endpoints form a dense subset of the unit circle. This implies the "if" part.

Remark 1. From Theorem 4, for any $f \in \mathcal{F}, \iota_{n, f}=\max _{w_{n}}\left|I_{w_{n}}\right|$ tends to zero exponentially as $n$ goes to infinity.

\section{Martingales in probability theory}

In this section, we give a brief review of the theory of martingales in probability theory. The standard reference which we used is [22]. Suppose that we have a probability space $(\Omega, \mathcal{B}, P)$ consisting of a space $\Omega$, a $\sigma$-field $\mathcal{B}$ of subsets of $\Omega$, and a probability measure $P$ on the $\sigma$-field $\mathcal{B}$.

Definition 5. A random variable or measurable function with respect to the $\sigma$-field $\mathcal{B}$ is a map $f: \Omega \rightarrow \mathbf{R}$, i.e., a real-valued function $f(w)$ on $\Omega$, such that for every Borel set $B \subset \mathbf{R}, f^{-1}(B) \in \mathcal{B}$. 
Definition 6. Suppose $\mathcal{B}^{\prime}$ is a sub- $\sigma$-field of $\mathcal{B}$. Suppose that $f$ and $g$ are $L^{1}$ integrable random variables with respect to $\mathcal{B}$ and $\mathcal{B}^{\prime}$ respectively. We call $g$ the conditional expectation of $f$ for the given sub- $\sigma$-field $\mathcal{B}^{\prime}$ and denote it by $g=E\left[f \mid \mathcal{B}^{\prime}\right]$ if

for all $A \in \mathcal{B}^{\prime}$.

$$
\int_{A} g(w) d P=\int_{A} f(w) d P
$$

The Radon-Nikodym theorem shows the existence of conditional expectation for any $L^{1}$ integrable random variable with respect to $\mathcal{B}$ for any given sub- $\sigma$-field of $\mathcal{B}$ (refer to [22]). The conditional expectation is unique up to measure zero set (also refer to [22]).

Definition 7. Suppose $(\Omega, \mathcal{B}, P)$ is a probability space. Suppose that $\left\{\mathcal{B}_{n}\right\}_{n=1}^{\infty}$ is a sequence of sub- $\sigma$-fields of $\mathcal{B}$ and suppose that $\left\{X_{n}\right\}_{n=1}^{\infty}$ is a sequence of functions. Then $\left\{\left(X_{n}, \mathcal{B}_{n}\right)\right\}_{n=1}^{\infty}$ is called an infinite martingale sequence if

(1) $X_{n}$ is a $L^{1}$ integrable random variable with respect to $\mathcal{B}_{n}$ for all $n \geq 1$;

(2) $\mathcal{B}_{n} \subset \mathcal{B}_{n+1}$ for all $n \geq 1$;

(3) $X_{n}=E\left[X_{n+1} \mid \mathcal{B}_{n}\right]$ almost everywhere with respect to the probability measure $P$.

An infinite martingale sequence $\left\{\left(X_{n}, \mathcal{B}_{n}\right)\right\}_{n=1}^{\infty}$ is called uniformly integrable if there is a constant $C>0$ such that

$$
\int_{\Omega} X_{n}(w) d P \leq C, \quad \forall n \geq 1 .
$$

Theorem 5. (Martingale Convergence Theorem) Suppose $\left\{\left(X_{n}, \mathcal{B}_{n}\right)\right\}_{n=1}^{\infty}$ is an uniformly integrable infinite martingale sequence. Then there is a random variable $X$ with respect to $\mathcal{B}$ such that

$$
X_{n}=E\left[X \mid B_{n}\right],
$$

and then, of course, $X_{n} \rightarrow X$ in $L^{1}$ as $n$ goes to $\infty$.

The main purpose of this paper is to construct a uniformly bounded infinite martingale sequence $\left\{\left(X_{n}, \mathcal{B}_{n}\right)\right\}_{n=1}^{\infty}$ from a uniformly quasisymmetric circle endomorphism $f$ and then apply the above theorem to construct the limiting martingale $X$.

\section{Dual symbolic representation and quasisymmetric invariant probability measures}

Suppose $f$ is a circle endomorphism in $\mathcal{F}$. Suppose

$$
\varpi_{n}=\left\{J_{w_{n}} \mid w_{n}=i_{0} i_{1} \cdots i_{n-1}, i_{k} \in\{0,1, \cdots, d-1\}, k=0,1, \cdots, n-1\right\},
$$

for $n=0,1, \cdots$, and

$$
\eta_{n}=\left\{I_{w_{n}} \mid w_{n}=i_{0} i_{1} \cdots i_{n-1}, i_{k} \in\{0,1, \cdots, d-1\}, k=0,1, \cdots, n-1\right\},
$$

for $n=0,1, \cdots$, are the corresponding sequences of nested Markov partitions on $T$ and $[0,1]$, respectively.

For any $w_{n}=i_{0} i_{1} \cdots i_{n-1}$, let

$$
w_{n}^{*}=j_{n-1} j_{n-2} \cdots j_{0},
$$


where $j_{n-1}=i_{0}, \cdots, j_{0}=i_{n-1}$.

For any fixed $w_{n}^{*}=j_{n-1} j_{n-2} \cdots j_{0}$ and any $n \geq 0$, define the right cylinder

$$
\left[w_{n}^{*}\right]=\left\{w^{*}=\cdots j_{n}^{\prime} j_{n-1}^{\prime} j_{n-2}^{\prime} \cdots j_{0}^{\prime} \mid j_{n-1}^{\prime}=j_{n-1}, j_{n-2}^{\prime}=j_{n-2}, \cdots, j_{0}^{\prime}=j_{0}\right\} .
$$

Define

$$
\Omega=\prod_{-\infty}^{0}\{0, \cdots, d-1\}=\left\{w^{*}=\cdots j_{n-1} \cdots j_{0}\right\} .
$$

For any fixed $w_{n}^{*}=j_{n-1} j_{n-2} \ldots j_{0}$ and any $n \geq 0$, define the right cylinder

$$
\left[w_{n}^{*}\right]=\left\{w^{*}=\ldots j_{n-1}^{\prime} j_{n-2}^{\prime} j_{n-2}^{\prime} \ldots j_{0}^{\prime} \mid j_{n-1}^{\prime}=j_{n-1}, j_{n-2}^{\prime}=j_{n-2}, \ldots, j_{0}^{\prime}=j_{0}\right\} .
$$

Consider

$$
\mathcal{G}=\left\{\bigcup_{k=1}^{N}\left[w_{n_{k}}^{*}\right]\right\} \cup\{\emptyset\} .
$$

The following proposition is easy.

Proposition 1. If $n<m$, then either $\left[w_{n}^{*}\right] \cap\left[w_{m}^{*}\right]=\emptyset$ or $\left[w_{n}^{*}\right] \cap\left[w_{m}^{*}\right]=\left[w_{m}^{*}\right]$.

Following this, we have that

Proposition 2. The set $\mathcal{G}$ is a field.

Proof. From Property 1, we can assume every element in $\mathcal{G}$ is a disjoint union of finitely many right cylinders $\left\{\left[w_{n_{k}}^{*}\right]\right\}_{k=1}^{N}$.

First

$$
\Omega=\bigcup_{k=0}^{d-1}\left[k^{*}\right] \in \mathcal{G}
$$

and $\emptyset \in \mathcal{G}$.

If $A, B \in \mathcal{G}$, then $A \cup B$ and $A \cap B$ are in $\mathcal{G}$. If $w_{n}^{*}=j_{n-1} j_{n-2} \ldots j_{0}$, then

$$
\left[w_{n}^{*}\right]^{c}=\bigcup_{0 \leq k \neq j_{n-1} \leq d-1}\left[k w_{n-1}^{*}\right] \bigcup \bigcup_{\widetilde{w}_{n-1}^{*} \neq w_{n-1}^{*}}\left[\widetilde{w}_{n-1}^{*}\right] \in \mathcal{G}
$$

and

$$
\left(\bigcup_{k=1}^{N}\left[w_{n_{k}}^{*}\right]\right)^{c}=\bigcap_{k=1}^{N}\left[w_{n_{k}}^{*}\right]^{c} \in \mathcal{G} .
$$

Thus $\mathcal{G}$ is a field.

Let $\mathcal{B}$ be the $\sigma$-field generated by the field $\mathcal{G}$. Then we have a space

$$
(\Omega, \mathcal{B}) \text {. }
$$

Now we are going to associate a non-atomic probability measure $P_{f}$ on this space with some uniformly quasisymmetric circle endomorphism $f$. Before we are able to do this, we need to have the following important invariant condition. We use Leb $(\cdot)$ to denote the Lebesgue measure on $T$.

Definition 8. We say that a circle endomorphism $f$ preserves the Lebesgue measure if for any Lebesgue measurable set $A$ in the unit circle $T$,

$$
\operatorname{Leb}\left(f^{-1}(A)\right)=\operatorname{Leb}(A) .
$$


This is equivalent to the following condition, for any interval $I$ in the unit interval $[0,1]$

$$
\sum_{k=0}^{d-1}\left|F^{-1}(I+k)\right|=|I| .
$$

One easy example of a circle endomorphism preserves the Lebesgue measure is $q(z)=z^{d}$ for any $d>1$. Actually, there are many circle endomorphisms preserve the Lebesgue measure. A well-known example among experts is a Blaschke product fixing zero and mapping the unit disk onto itself (for example, see [18]). More precisely, consider the Blaschke product

$$
f(z)=z^{j} \prod_{1 \leq i \leq d-j} \frac{z-a_{i}}{1-\bar{a}_{i} z}: T \rightarrow T
$$

where $1 \leq j \leq d$ and $\left|a_{i}\right|<1,1 \leq i \leq d-j$. Then it is a circle endomorphism of degree $d>1$.

Example 2. Every $f$ in the form of (8) preserves the Lebesgue measure.

Proof. For reader's convenience, we include a detailed proof. The equality (6) is equivalent to

$$
\int_{T} \phi(f(z)) d z=\int_{T} \phi(z) d z
$$

for all continuous functions $\phi$ on $T$.

Now let $\phi$ be a continuous function on $T$. Consider the harmonic partial differential equation on the unit disk $D=\{z \in \mathbf{C}|| z \mid<1\}$ :

$$
\left\{\begin{array}{l}
\Delta u=0 \\
u \mid T=\phi
\end{array}\right.
$$

Then it has a unique solution $u$ which is a harmonic function on $D$. Since $f$ is analytic on $\mathbf{C}, u \circ f$ is also a harmonic function on $D$ with a continuous extension to the boundary $T$ of $D$. By the mean value theorem in harmonic analysis,

$$
\int_{T} \phi(f(z)) d z=2 \pi i u(f(0)) \text { and } \int_{T} \phi(z) d z=2 \pi i u(0) .
$$

But $f(0)=0$, so we verified the equality (9) is true.

Remark 2. Actually, there are much more circle endomorphisms preserving the Lebesgue measure. It is known that for any $C^{1+\alpha}$ expanding circle endomorphism $\tilde{f}$, there is a $C^{1+\alpha}$-diffeomorphism $h$ of $T$ such that $f=h \circ \tilde{f} \circ h^{-1}$ preserves the Lebesque measure (see, for example, [16]).

Remark 3. In addition, a circle endomorphism $f$ is called uniformly symmetric if there is a bounded function $\epsilon(t)>0$ such that $\epsilon(t) \rightarrow 0$ as $t \rightarrow 0^{+}$and such that

$$
\frac{1}{1+\epsilon(t)} \leq \frac{\left|F^{-n}(x+t)-F^{-n}(x)\right|}{\left|F^{-n}(x)-F^{-n}(x-t)\right|} \leq 1+\epsilon(t)
$$

for all $x \in \mathbf{R}$ and $t>0$ and any $n>0$. A $C^{1+\alpha}$ expanding circle endomorphism is uniformly symmetric (see $[19,17]$ ). It is clear that a uniformly symmetric circle endomorphism is uniformly quasisymmetric. It has been proved in [19] that for any 
uniformly symmetric circle endomorphism $\tilde{f}$, there is a symmetric homeomorphism $h$ of $T$ with $h(1)=1$ such that $f=h \circ \widetilde{f} \circ h^{-1}$ is still uniformly symmetric and preserves the Lebesgue measure.

Remark 4. However, for arbitrary uniformly quasisymmetric circle endomor$\operatorname{phism} \tilde{f}$, we do not have a similar statement to Remark 3 . The reason is that if $f$ preserves the Lebesgue measure then the local quasiconformal dilatations of $f$ at the grand orbit

$$
G O(x)=\bigcup_{m=0}^{\infty} \bigcup_{n=0}^{\infty} f^{-n}\left(f^{m}(x)\right)
$$

of any point $x$ must be the same. This property will not be changed by conjugating by a symmetric circle homeomorphism. But it is easy to construct a counter-example $f$ of a uniformly quasisymmetric circle endomorphism such that the local quasiconformal dilatations at $x$ and one point of $f^{-1}(x)$ are different. So the following problem is interesting for us. Find conditions on a uniformly quasisymmetric circle endomorphism $\tilde{f}$ such that there is a symmetric circle homeomorphism $h$ such that $f=h \circ \tilde{f} \circ h^{-1}$ preserves the Lebesgue measure. (Refer to $[19,17]$ for the relevant materials to study this problem.)

Suppose $f \in \mathcal{F}$ preserves the Lebesgue measure. We define a $P=P_{f}$ on $\mathcal{G}$ as

$$
P(\emptyset)=0, \quad P\left(\left[w_{n}^{*}\right]\right)=\left|I_{w_{n}^{*}}\right|, \quad \text { and } \quad P\left(\bigcup_{k=1}^{N}\left[w_{n_{k}}^{*}\right]\right)=\sum_{k=1}^{N}\left|I_{w_{n_{k}}^{*}}\right|,
$$

where $\bigcup_{k=1}^{N}\left[w_{n_{k}}^{*}\right]$ is a disjoint union. Then $P(\Omega)=\left|I_{0}\right|+\left|I_{1}\right| \cdots+\left|I_{d-1}\right|=1$.

For any right cylinder $\left[w_{n}^{*}\right],\left[k w_{n}^{*}\right], k=0, \cdots, d-1$, are all the sub-cylinders of one level lower and

$$
\left[w_{n}^{*}\right]=\bigcup_{k=0}^{d-1}\left[k w_{n}^{*}\right]
$$

This implies that

$$
f^{-1}\left(J_{w_{n}^{*}}\right)=\bigcup_{k=0}^{d-1} J_{k w_{n}^{*}}
$$

Since $f$ preserves the Lebesgue measure, we have that

$$
\left|I_{w_{n}^{*}}\right|=\sum_{k=0}^{d-1}\left|I_{k w_{n}^{*}}\right|
$$

This implies that

$$
P\left(\left[w_{n}^{*}\right]\right)=\sum_{k=0}^{d-1} P\left(\left[k w_{n}^{*}\right]\right) .
$$

So $P$ is a probability measure on $\mathcal{G}$. This fact plus the Kolmogorov extension principle (or the Carathéodory Theorem), $P$ can be extended to a probability measure on the $\sigma$-field $\mathcal{B}$, which we still denote as $P_{f}$ or simply $P$ if there is no confusion. The probability measure $P$ is non-atomic. Thus we construct a probability space

$$
(\Omega, \mathcal{B}, P)
$$

for every $f \in \mathcal{F}$ preserving the Lebesgue measure. 
Define a right shift map $\sigma^{*}$ as

$$
\sigma^{*}\left(w_{n}^{*}\right)=j_{n-1} j_{n-2} \cdots j_{1}
$$

for $w_{n}^{*}=j_{n-1} j_{n-2} \cdots j_{1} j_{0}$. It is extended to the right shift, we still denote as $\sigma^{*}: \Omega \rightarrow$ $\Omega$, as

$$
\sigma^{*}\left(w^{*}\right)=\cdots j_{n-1} j_{n-2} \cdots j_{1}
$$

for $w^{*}=\cdots j_{n-1} j_{n-2} \cdots j_{1} j_{0}$.

Define the adding machine as

$$
\operatorname{add}\left(j_{n-1} \cdots j_{1} j_{0}\right)=j_{n-1} \cdots\left(j_{k}+1\right) 0 \cdots 0
$$

if $0 \leq k<n-1$ is the first integer such that $0 \leq j_{k}<d-1$ and

$$
\operatorname{add}((d-1) \cdots(d-1))=0 \cdots 0 .
$$

From Theorem 4, we have the following result.

Theorem 6. The probability measure $P$ is $\sigma^{*}$-invariant, that is,

$$
P\left(\left(\sigma^{*}\right)^{-1}(A)\right)=P(A)
$$

for any $A \in \mathcal{B}$. Moreover, there is a constant $C>0$ such that

$$
C^{-1} \leq \frac{P\left(\left[w_{n}^{*}\right]\right)}{P\left(\operatorname{add}\left(\left[w_{n}^{*}\right]\right)\right)} \leq C
$$

for all $w_{n}^{*}=j_{n-1} \cdots j_{1} j_{0}$.

Following the proof of Theorem 4 and the construction of $P_{f}$, we have also that

Theorem 7. For any non-atomic $\sigma^{*}$-invariant probability measure $P$ on $(\Omega, \mathcal{B})$ satisfying the condition (10), there is an $f \in \mathcal{F}$ preserving the Lebesgue measure such that $P_{f}=P$.

Define the space

$$
\mathcal{F}_{\text {inv }}=\{f \in \mathcal{F} \mid f \text { preserves the Lebesgue measure }\} .
$$

Then the corresponding space in $\mathcal{H}$ is

$$
\mathcal{H}_{\text {inv }}=\left\{h \in \mathcal{H} \mid \operatorname{Leb}\left(h\left(q^{-1}(A)\right)\right)=\operatorname{Leb}(h(A)) \text { for any measurable set } A\right\} .
$$

The Leb-invariant condition in the definition of $\mathcal{H}_{\text {inv }}$ can be translated into the following condition for the lift $H$ of $h$ :

$$
\sum_{k=0}^{k=d-1}\left(H\left(\frac{x+k}{d}\right)-H\left(\frac{k}{d}\right)\right)=H(x) .
$$

Define the probability measure space

$$
\begin{gathered}
\mathcal{M}_{\text {inv }}=\left\{P \mid P \text { is a non-atomic } \sigma^{*} \text {-invariant probability measure } P \text { on }(\Omega, \mathcal{B})\right. \\
\text { satisfying the condition }(10)\} .
\end{gathered}
$$

We call a measure in $\mathcal{M}_{\text {inv }}$ a quasisymmetric invariant probability measure.

Then

is bijective. Define

$$
\beta: \mathcal{H}_{\text {inv }} \rightarrow \mathcal{F}_{\text {inv }}
$$

$$
\gamma: \mathcal{F}_{\text {inv }} \rightarrow \mathcal{M}_{\text {inv }}
$$


by $\gamma(f)=P_{f}$. Then it is also surjective. We are still interested in the injectivity of $\gamma$. This is equivalent to the following conjecture.

Conjecture 1. Given any two $f, g \in \mathcal{F}_{\text {inv }}$. Then $f=g$ if and only if there is a sequence $\epsilon(n) \rightarrow 0$ as $n \rightarrow \infty$ such that

$$
\left|\frac{P_{f}\left(\left[w_{n}^{*}\right]\right)}{P_{f}\left(\operatorname{add}\left(\left[w_{n}^{*}\right]\right)\right)}-\frac{P_{g}\left(\left[w_{n}^{*}\right]\right)}{P_{g}\left(\operatorname{add}\left(\left[w_{n}^{*}\right]\right)\right)}\right| \leq \epsilon(n)
$$

for all $n>0$.

A related conjecture is that

Conjecture 2. Suppose $f, g \in \mathcal{F}_{\text {inv }}$ are conjugate by a symmetric homeomorphism $h$, that is, $h \circ f=g \circ h$. Then $h$ must be the identity.

The reader who is interested in Conjecture 2 can refer to [18] for the proof of this conjecture under the smooth assumption and for the proof of this conjecture under the assumption that one of $f$ and $g$ is $z \mapsto z^{d}$. The reader who is interested in the equivalence between Conjecture 1 and Conjecture 2 can refer to [5] for some metric property of symmetric homeomorphisms.

\section{Martingales revisited}

For any fixed $n \geq 0$, define $\mathcal{B}_{n}$ as the $\sigma$-field generated by all right cylinders $\left\{\left[w_{s}^{*}\right], 0 \leq s \leq n\right\}$. Then $\mathcal{B}_{n}$ is a sub- $\sigma$-field and we have a filter

$$
\cdots \subset \mathcal{B}_{n} \subset \mathcal{B}_{n+1} \subset \cdots \subset \mathcal{B} \text {. }
$$

Consider $P=P_{f}$ for any $f \in \mathcal{F}_{\text {inv }}$. Define

and define

$$
X_{n}\left(w^{*}\right)=\frac{P\left(\left[\sigma^{*}\left(w_{n}^{*}\right)\right]\right)}{P\left(\left[w_{n}^{*}\right]\right)}
$$

$$
B X_{n}\left(w^{*}\right)=\frac{P\left(\left[\operatorname{add}\left(w_{n}^{*}\right)\right]\right)}{P\left(\left[w_{n}^{*}\right)\right)}
$$

for any $w^{*}=\cdots w_{n}^{*} \in\left[w_{n}^{*}\right]$. Then $\left\{X_{n}\right\}_{n=0}^{\infty}$ and $\left\{B X_{n}\right\}_{n=0}^{\infty}$ are two sequences of random variables defined on $\Omega$. We have the following theorem.

Theorem 8. Suppose $f$ is a uniformly quasisymmetric circle endomorphism preserving the Lebesgue measure. Both sequences $\left\{\left(X_{n}, \mathcal{B}_{n}\right)\right\}_{n=0}^{\infty}$ and $\left\{\left(B X_{n}, \mathcal{B}_{n}\right)\right\}_{n=0}^{\infty}$ are uniformly bounded infinite martingale sequences and bounded away from 0 uniformly. Therefore, there are two bounded $L^{1}$ functions $X(w)$ and $B X(w)$ bounded away from 0 defined on $(\Omega, \mathcal{B}, P)$ such that

$$
X_{n}\left(w^{*}\right) \rightarrow X\left(w^{*}\right) \quad \text { and } \quad B X_{n}\left(w^{*}\right) \rightarrow B X\left(w^{*}\right)
$$

in the $L^{1}$-norm.

Proof. First, on every right cylinder $\left[w_{n}^{*}\right]$,

$$
X_{n}\left(w^{*}\right)=\frac{P\left(\left[\sigma^{*}\left(w_{n}^{*}\right)\right]\right)}{P\left(\left[w_{n}^{*}\right]\right)}=\frac{\left|I_{\sigma^{*}\left(w_{n}^{*}\right)}\right|}{\left|I_{w_{n}^{*}}\right|}
$$

is a constant. So $\left\{X_{n}\left(w^{*}\right) \leq x\right\}$ is the union of some open set $\left[w_{n_{k}} *\right] \in \mathcal{B}_{n}$ on which the function $X_{n}\left(w^{*}\right)=\frac{\left|I_{\sigma^{*}\left(w_{n_{k}}^{*}\right)}\right|}{\left|I_{w_{n_{k}}^{*}}\right|}$ is a constant less than or equal to $x$ for every $k$. Hence $X_{n}\left(w^{*}\right)$ is $\mathcal{B}_{n}$-measurable. 


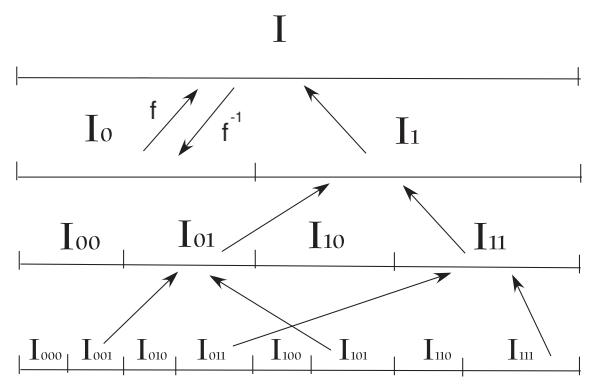

Markov partition of the unit interval for a degree 2 map $f(z)$.

Figure 2.

Second, $X_{n}\left(w^{*}\right)$ is uniformly integrable as follows. The expectation $E\left[X_{n}\right]=\sum_{w_{n}^{*}} \frac{\left|I_{\sigma^{*}\left(w_{n}^{*}\right)}\right|}{\left|I_{w_{n}^{*}}\right|} \cdot P\left(\left[w_{n}^{*}\right]\right)=\sum_{w_{n}^{*}} \frac{\left|I_{\sigma^{*}\left(w_{n}^{*}\right)}\right|}{\left|I_{w_{n}^{*}}\right|} \cdot\left|I_{w_{n}^{*}}\right|=\sum_{w_{n}^{*}}\left|I_{\sigma^{*}\left(w_{n}^{*}\right)}\right| \leq d<\infty$.

Third, we have the following important relation for the conditional expectation $E\left[X_{n} \mid \mathcal{B}_{n-1}\right]$ :

$$
X_{n-1}=E\left[X_{n} \mid \mathcal{B}_{n-1}\right]
$$

almost everywhere with respect to $\mathrm{P}$. That is equivalent to say that

$$
\int_{\left[w_{n-1}^{*}\right]} X_{n-1}\left(w^{*}\right) d P\left(w^{*}\right)=\int_{\left[w_{n-1}^{*}\right]} X_{n}\left(w^{*}\right) d P\left(w^{*}\right)
$$

for every right cylinder $\left[w_{n-1}^{*}\right] \in \mathcal{B}_{n-1}$. The reason is as follows.

Let

$$
R H S=\int_{\left[w_{n-1}^{*}\right]} X_{n}\left(w^{*}\right) d P\left(w^{*}\right) \quad \text { and } \quad L H S=\int_{\left[w_{n-1}^{*}\right]} X_{n-1}\left(w^{*}\right) d P\left(w^{*}\right) .
$$

Then

$$
R H S=\int_{\left[w_{n-1}^{*}\right]} \frac{\left|I_{\sigma^{*}\left(w_{n}^{*}\right)}\right|}{\left|I_{w_{n}^{*}}\right|} d P\left(w^{*}\right)=\sum_{k=0}^{d-1} \int_{\left[k w_{n-1}^{*}\right]} \frac{\left|I_{\sigma^{*}\left(w_{n}^{*}\right)}\right|}{\left|I_{w_{n}^{*}}\right|} d P\left(w^{*}\right) .
$$

So

$$
R H S=\sum_{k=0}^{d-1} \frac{\left|I_{\sigma^{*}\left(k w_{n-1}^{*}\right)}\right|}{\left|I_{k w_{n-1}^{*}}\right|} \cdot\left|I_{k w_{n-1}^{*}}\right|=\sum_{k=0}^{d-1}\left|I_{\sigma^{*}\left(k w_{n-1}^{*}\right)}\right|=\left|I_{\sigma^{*}\left(w_{n-1}^{*}\right)}\right| .
$$

The last equality is because $f$ preserves the Lebesgue measure.

Now

$$
L H S=\int_{\left[w_{n-1}^{*}\right]} \frac{\left|I_{\sigma^{*}\left(w_{n-1}^{*}\right)}\right|}{\left|I_{w_{n-1}^{*}}\right|} d P\left(w^{*}\right)=\frac{\left|I_{\sigma^{*}\left(w_{n-1}^{*}\right)}\right|}{\left|I_{w_{n-1}^{*}}\right|} \cdot\left|I_{w_{n-1}^{*}}\right|=\left|I_{\sigma^{*}\left(w_{n-1}^{*}\right)}\right| .
$$

We got that $R H S=L H S$.

From the bounded nearby geometry in $\S 4$, we have constants $0<m<M$ such that

$$
m<X_{n}\left(w^{*}\right) \leq M
$$

for all $w^{*} \in \Omega$ and $n \geq 0$. Therefore, $\left\{\left(X_{n}, \mathcal{B}_{n}\right)\right\}_{n=0}^{\infty}$ is a bounded martingale and bounded away from 0 uniformly. 
From the martingale convergence theorem, we have a bounded $L^{1}$ function $X\left(w^{*}\right)$ on the probability space $(\Omega, \mathcal{B}, P)$ such that

$$
X_{n}\left(w^{*}\right) \rightarrow X\left(w^{*}\right)
$$

in the $L^{1}$-norm. We completed the proof for $\left\{\left(X_{n}, \mathcal{B}_{n}\right)\right\}_{n=0}^{\infty}$.

The proof for $\left\{\left(B X_{n}, \mathcal{B}_{n}\right)\right\}_{n=0}^{\infty}$ is similar. We leave it as an exercise for the reader.

We call the random variable (or function) $X\left(w^{*}\right)$ in the above theorem a limiting martingale and the random variable (or function) $B X\left(w^{*}\right)$ in the above theorem a limiting nearby martingale.

Remark 5. In Theorem 8, the condition $f$ being uniformly quasisymmetric implies that the corresponding Markov partition has bounded nearby geometry. The bounded nearby geometry implies the bounded geometry. However the other direction doesn't work (see the following example). Note that, for the existence of $X\left(w^{*}\right)$, the Morkov partition only needs to have bounded geometry which is a weaker condition.

Example 3. Assume $F(x)=3 x$ for $x \in\left[0, \frac{1}{3}\right]$ and $F(x)=\frac{3}{2}\left(x-\frac{1}{3}\right)$ for $x \in\left[\frac{1}{3}, 1\right]$. Then $F(x)$ has bounded geometry, but not bounded nearby geometry.

Remark 6. If $\tilde{f}$ is a $C^{1+\alpha}$ expanding map, then it is uniformly quasisymmetric (see [19]) and there is a unique $C^{1+\alpha}$-diffeomorphism $h$ of $T$ with $h(1)=1$ such that $f=h \circ \widetilde{f} \circ h^{-1}$ preserves the Lebesgue measure (see Remark 3) and the martingales $\left\{\left(X_{n}, \mathcal{B}_{n}\right)\right\}_{n=0}^{\infty}$ and $\left\{\left(B X_{n}, \mathcal{B}_{n}\right)\right\}_{n=0}^{\infty}$ induced from $f$ converge to the limiting martingale $X\left(w^{*}\right)$ and the limiting nearby martingale $B X\left(w^{*}\right)$ exponentially. That is, there is a constant $C>0$ and $0<\tau<1$ such that

$$
\max _{w^{*} \in \Omega}\left|X_{n}\left(w^{*}\right)-X\left(w^{*}\right)\right| \leq C \tau^{n}, \quad \forall n \geq 0
$$

and

$$
\max _{w^{*} \in \Omega}\left|B X_{n}\left(w^{*}\right)-B X\left(w^{*}\right)\right| \leq C \tau^{n}, \quad \forall n \geq 0 .
$$

Moreover, the limiting martingale $X\left(w^{*}\right)$ and the limiting nearby martingale $B X\left(w^{*}\right)$ are Hölder continuous functions in the meaning that there is a constant $C>0$ and $0<\tau<1$ such that

$$
\left|X\left(w^{*}\right)-X\left(\widetilde{w}^{*}\right)\right| \leq C \tau^{n} \quad \text { and } \quad\left|B X\left(w^{*}\right)-B X\left(\widetilde{w}^{*}\right)\right| \leq C \tau^{n}
$$

for all $w^{*}=\cdots j_{n} w_{n}^{*}$ and $\widetilde{w}^{*}=\cdots \widetilde{j}_{n} w_{n}^{*}$ and $w_{n}^{*}=j_{n-1} \cdots j_{0}$. In [19], $X\left(w^{*}\right)$ is called the dual derivative and is used in the study of dual Gibbs measure theory for Hölder continuous potentials. The reader who is interested in this result can go to [19].

Remark 7. The space $\Omega$ is also a metric space with a standard metric

$$
d\left(w^{*}, \widetilde{w}^{*}\right)=\sum_{n=1}^{\infty} \frac{\left|j_{n-1}-\widetilde{j}_{n-1}\right|}{d^{n}}
$$

where $w^{*}=\cdots j_{n-1} \cdots j_{0}$ and $\widetilde{w}^{*}=\cdots \widetilde{j}_{n-1} \cdots \widetilde{j}_{0}$. Suppose $\tilde{f}$ is a uniformly symmetric circle endomorphism (see Remark 3 ). Then there is a symmetric homeomorphism $h$ of $T$ with $h(1)=1$ such that $f=h \circ \tilde{f} \circ h^{-1}$ preserves the Lebesgue measure (see [19]) and the martingales $\left\{X_{n}, \mathcal{B}_{n}\right\}_{n=0}^{\infty}$ and $\left\{B X_{n}, \mathcal{B}_{n}\right\}_{n=0}^{\infty}$ induced from $f$ converge to the 
limiting martingale $X\left(w^{*}\right)$ the limiting nearby martingale $B X\left(w^{*}\right)$ uniformly with respect to the metric $d(\cdot, \cdot)$. That is,

$$
\max _{w^{*} \in \Omega}\left|X_{n}\left(w^{*}\right)-X\left(w^{*}\right)\right| \rightarrow 0, \quad \text { as } n \rightarrow \infty
$$

and

$$
\max _{w^{*} \in \Omega}\left|B X_{n}\left(w^{*}\right)-B X\left(w^{*}\right)\right| \rightarrow 0, \quad \text { as } n \rightarrow \infty .
$$

Moreover, the limiting martingale $X\left(w^{*}\right)$ and the limiting nearby martingale $B X\left(w^{*}\right)$ are continuous functions. In [19], $X\left(w^{*}\right)$ is also called the dual derivative and is used in the study of dual Gibbs measure theory for continuous potentials. The reader who is interested in this result can go to [19].

Remark 8. The limiting martingale $X$ and the limiting nearby martingale $B X$ are symmetric invariants. This means that if $f_{1}$ and $f_{2}$ are both uniformly quasisymmetric circle endomorphisms and conjugated by a symmetric homeomorphism $h$, that is, $f_{1}=h \circ f_{2} \circ h^{-1}$ and if $X_{1}$ and $X_{2}$ are limiting martingales and $B X_{1}$ and $B X_{2}$ are limiting nearby martingales, then $X_{1}=X_{2}$ and $B X_{1}=B X_{2}$. Actually, in [17] (see also [19]), it has been proved that

$$
\lim _{n \rightarrow \infty}\left\|X_{n, 1}-X_{n, 2}\right\|=0 \quad \text { and } \quad \lim _{n \rightarrow \infty}\left\|B X_{n, 1}-B X_{n, 2}\right\|=0
$$

for any circle endomorphisms $f_{1}$ and $f_{2}$ conjugated by a symmetric circle homeomorphism $h$, does not matter the limits exist or not for $X_{n, 1}$ or $X_{n, 2}$ or $B X_{n, 1}$ or $B X_{n, 2}$, where $\|\cdot\|$ means the maximum norm.

Concluding from this section, we proved that $\mathcal{F}_{\text {inv }}$ is the space where we can define limiting martingales and limiting nearby martingales on the space $(\Omega, \mathcal{B})$. If two maps in $\mathcal{F}_{\text {inv }}$ are symmetrically conjugate, then their limiting martingales are the same and their limiting nearby martingales are the same. Define $\mathcal{M T}$ as the space of all limiting martingales $X_{f}$ with the probability measure $P_{f}$ for $f \in \mathcal{F}_{\text {inv }}$ and define $\mathcal{B} M T$ as the space of all limiting nearby martingales $B X_{f}$ with the probability measure $P_{f}$ for $f \in \mathcal{F}_{\text {inv }}$. We are still interested in the following problem.

Problem 1. Given a characterization of $X_{f}$ for any $f \in \mathcal{F}_{\text {inv }}$ such that for any $P \in \mathcal{M}_{\text {inv }}$ and any $L^{1}$ function $X$ with respect to $(\Omega, \mathcal{B}, P)$ satisfying this characterization, there is an $f \in \mathcal{F}_{\text {inv }}$ such that $X_{f}=X$.

Furthermore, we have the following conjecture.

Conjecture 3. Suppose the limiting martingales or the limiting nearby martingales corresponding to $f_{1}$ and $f_{2}$ in $\mathcal{F}_{\text {inv }}$ are the same, that is, $X_{1}=X_{2}$ and $P_{1} \sim P_{2}$ or $B X_{1}=B X_{2}$ and $P_{1} \sim P_{2}$ where $P_{1} \sim P_{2}$ means that $P_{1}$ is absolutely continuous with respect to $P_{2}$ and $P_{2}$ is absolutely continuous with respect to $P_{1}$. Then $f_{1}=f_{2}$.

This conjecture is closely related to Conjecture 1 .

Remark 9. If both of $f_{1}$ and $f_{2}$ are $C^{1+\alpha}, 0<\alpha \leq 1$, expanding circle endomorphisms and preserving the Lebesgue measure, it has been proved in $[14,15,18]$ that if the limiting martingales or the limiting nearby martingales corresponding to $f_{1}$ and $f_{2}$ are the same, that is, $X_{1}=X_{2}$ or $B X_{1}=B X_{2}$, then $f_{1}$ and $f_{2}$ are smoothly conjugate. More precisely, there is a $C^{1+\alpha}$ diffeomorphism $h$ of $T$ such that

$$
f_{1}=h \circ f_{2} \circ h^{-1} \text {. }
$$

Furthermore, $h$ is the identity. Thus $f_{1}=f_{2}$ and $P_{1}=P_{2}$. 
When $f_{1}$ and $f_{2}$ are both uniformly symmetric circle endomorphisms, the reader can refer to $[19,17,18]$ for some related results.

\section{Hilbert transform and almost complex structure}

In the rest of the paper, we would like to discuss an almost complex structure and complex manifold structure on $\mathcal{F}_{\text {inv }}$ (as well as $\mathcal{H}_{\text {inv }}$ ). The relation between the classical Hilbert transform and the complex structure on the universal Teichmüller space has been studied deeply (refer to $[8,4,20]$ ). We will use this idea to study an almost complex structure and complex structure on $\mathcal{F}_{\text {inv. }}$. In this section, we will prove that the Hilbert transform defines a natural almost complex structure on $\mathcal{F}_{\text {inv }}$.

The space $\mathcal{F}_{\text {inv }}$ is a subspace of $\mathcal{F}$. There is a bijective map $\beta$ from $\mathcal{H}$ to $\mathcal{F}$ such that it is also a bijective map from $\mathcal{H}_{\text {inv }}$ to $\mathcal{F}_{\text {inv }}$. We will prove that the Hilbert transform defined on $\mathcal{H}$ preserves the space $\mathcal{H}_{\text {inv }}$. Thus the Hilbert transform defines a natural almost complex structure on $\mathcal{H}_{\text {inv }}$ as well as on $\mathcal{F}_{\text {inv }}$.

Following the study of the universal Teichmüller space (refer to [8]), the tangent space $\mathcal{V}$ of $\mathcal{H}_{\text {inv }}$ at the identity is the space of all Zygmund functions on the real line satisfying that

$$
V(0)=0 \quad \text { and } \quad V(x+1)=V(x)
$$

and

$$
\sum_{k=0}^{d-1}\left(V\left(\frac{x+k}{d}\right)-V\left(\frac{k}{d}\right)\right)=V(x) .
$$

Example 4. When the degree $d=2$,

$$
V(x)=\sum_{n=1}^{\infty} 2^{-n} \sin \left(2^{n} \pi x\right)
$$

is a vector in the tangent space $\mathcal{V}$ of $\mathcal{H}_{\text {inv }}$ since

$$
V(0)=0, \quad V(x+1)=V(x) \quad \text { and } \quad V\left(\frac{x}{2}\right)+V\left(\frac{1+x}{2}\right)-V\left(\frac{1}{2}\right)=V(x) .
$$

The Hilbert transform $\mathcal{J}$ on $\mathcal{V}$ is defined by

$$
\mathcal{J} V(x)=\frac{1}{\pi} \int_{-\infty}^{\infty} V(y) R(x, y) d y
$$

where

$$
R(x, y)=\frac{1}{y-x}-\frac{x}{y-1}+\frac{x-1}{y} .
$$

Let $\zeta=\xi+i \eta$ be a complex number. Then $\bar{\zeta}=\xi-i \eta$. By Stokes' formula,

$$
\mathcal{J} V(x)=\frac{1}{\pi} \int_{-\infty}^{\infty} V(\zeta) R(x, \zeta) d \zeta=\frac{2 i}{\pi} \iint_{\mathbf{H}} \bar{\partial} V(\zeta) R(x, \zeta) d \xi d \eta+i V(x),
$$

where $\mathbf{H}$ is the upper-half plane and $\bar{\partial} V=\partial V / \partial \bar{\zeta}$.

Let $\mu(\zeta)=\bar{\partial} V(\zeta)$, then

$$
\mathcal{J} V(x)=\frac{2 i}{\pi} \iint_{\mathbf{H}} \mu(\zeta) R(x, \zeta) d \xi d \eta+i V(x)=\frac{-2 i}{\pi} \iint_{\mathbf{L}} \mu(\zeta) R(x, \zeta) d \xi d \eta-i V(x),
$$

where $\mathbf{L}$ is the lower-half plane. 
Define

$$
\widetilde{\mu}(\zeta)= \begin{cases}-i \mu(\zeta), & \zeta \in \mathbf{H} \\ i \mu(\zeta), & \zeta \in \mathbf{L}\end{cases}
$$

Then

$$
\mathcal{J} V(x)=\frac{-1}{\pi} \iint_{\mathbf{C}} \widetilde{\mu}(\zeta) R(x, \zeta) d \xi d \eta
$$

And we also have

$$
\bar{\partial}(\mathcal{J} V)=-i \mu \quad \text { and } \quad \bar{\partial}\left(H^{2} V\right)=i^{2} \mu=-\mu .
$$

This implies

$$
\mathcal{J}^{2} V=-V
$$

Hence the Hilbert transform $\mathcal{J}$ gives an almost complex structure on the space of all Zygmund functions on the real line.

The following theorem shows that the Hilbert transform $\mathcal{J}$ also gives an almost complex structure on the space $\mathcal{H}_{\text {inv }}$. Let $\mathcal{I}$ denote the identity map on $\mathcal{H}_{\text {inv }}$.

Theorem 9. Let $\mathcal{J} V$ be the Hilbert transform of $V \in \mathcal{V}$, then $\mathcal{J} V$ satisfies the equations (12) and (13). Therefore, $\mathcal{J} V \in \mathcal{V}$. Since $\mathcal{J}: \mathcal{V} \rightarrow \mathcal{V}$ and $\mathcal{J}^{2}=-\mathcal{I}$, it is an almost complex structure on $\mathcal{H}_{\text {inv }}$.

Proof. Note that

$$
\mathcal{J} V(x)=\frac{1}{\pi} \int_{-\infty}^{\infty} V(y)\left(\frac{1}{y-x}-\frac{x}{y-1}+\frac{x-1}{y}\right) d y .
$$

From $V(x+1)=V(x)$, we have

$$
\frac{1}{\pi} \int_{-\infty}^{\infty} \frac{x V(y)}{y-1} d y=\frac{1}{\pi} \int_{-\infty}^{\infty} \frac{x V(y+1)}{y} d y=\frac{1}{\pi} \int_{-\infty}^{\infty} \frac{x V(y)}{y} d y
$$

Hence

$\mathcal{J} V(x)=\frac{1}{\pi} \int_{-\infty}^{+\infty} \frac{V(y)}{y-x} d y-\frac{1}{\pi} \int_{-\infty}^{\infty} \frac{V(y)}{y} d y=\frac{1}{\pi} \int_{-\infty}^{+\infty} \frac{V(y+x)}{y} d y-\frac{1}{\pi} \int_{-\infty}^{\infty} \frac{V(y)}{y} d y$.

From this form of the Hilbert transform, it is easy to check $\mathcal{J} V(0)=0$ and $\mathcal{J} V(x+$ $1)=\mathcal{J} V(x)$ since $V(y+1)=V(y)$. These are the equations in (12).

To prove the equation (13) for $\mathcal{J} V$, we only need to show

$$
\frac{1}{\pi} \int_{-\infty}^{+\infty} \frac{\sum_{k=0}^{d-1}\left(V\left(y+\frac{x+k}{d}\right)-V\left(y+\frac{k}{d}\right)\right)+V(y)-V(y+x)}{y} d y=0 .
$$

Since

$$
\sum_{k=0}^{d-1}\left(V\left(\frac{x+k}{d}\right)-V\left(\frac{k}{d}\right)\right)=V(x)
$$

we have that

$$
\sum_{k=0}^{d-1}\left(V\left(y+\frac{x+k}{d}\right)-V\left(\frac{k}{d}\right)\right)=V(y d+x)
$$

and

$$
\sum_{k=0}^{d-1}\left(V\left(y+\frac{k}{d}\right)-V\left(\frac{k}{d}\right)\right)=V(y d)
$$


The difference of these two equations is

$$
\sum_{k=0}^{d-1}\left(V\left(y+\frac{x+k}{d}\right)-V\left(y+\frac{k}{d}\right)\right)=V(y d+x)-V(y d) .
$$

Hence the numerator of the fraction inside the equation in the previous integral is

$$
\begin{aligned}
& \sum_{k=0}^{d-1}\left(V\left(y+\frac{x+k}{d}\right)-V\left(y+\frac{k}{d}\right)\right)+V(y)-V(y+x) \\
& =V(y)-V(y+x)+V(y d+x)-V(y d) .
\end{aligned}
$$

So we need to show

$$
\begin{aligned}
& \frac{1}{\pi} \int_{-\infty}^{+\infty} \frac{V(y)+V(y d+x)-V(y d)-V(y+x)}{y} d y \\
& =\frac{1}{\pi} \int_{-\infty}^{+\infty} \frac{V(y)-V(y d)}{y} d y+\frac{1}{\pi} \int_{-\infty}^{+\infty} \frac{V(y d+x)-V(y+x)}{y} d y=0 .
\end{aligned}
$$

It is easy to see that

$$
\frac{1}{\pi} \int_{-\infty}^{+\infty} \frac{V(y)}{y} d y=\frac{1}{\pi} \int_{-\infty}^{+\infty} \frac{V(y d)}{y d} d(y d)=\frac{1}{\pi} \int_{-\infty}^{+\infty} \frac{V(y d)}{y} d y
$$

So

$$
\frac{1}{\pi} \int_{-\infty}^{+\infty} \frac{V(y)-V(y d)}{y} d y=0
$$

For the term

$$
\frac{1}{\pi} \int_{-\infty}^{+\infty} \frac{V(y d+x)-V(y+x)}{y} d y
$$

we have

$$
\begin{aligned}
\frac{1}{\pi} \int_{-\infty}^{+\infty} \frac{V(y+x)}{y} d y & =\frac{1}{\pi} \int_{-\infty}^{+\infty} \frac{V(y)}{y-x} d y=\frac{1}{\pi} \int_{-\infty}^{+\infty} \frac{V(y d+x)}{y d} d(y d+x) \\
& =\frac{1}{\pi} \int_{-\infty}^{+\infty} \frac{V(y d+x)}{y} d y .
\end{aligned}
$$

Thus

$$
\frac{1}{\pi} \int_{-\infty}^{+\infty} \frac{V(y d+x)-V(y+x)}{y} d y=0 .
$$

We have proved the theorem.

\section{Complex manifold structure}

In this section, we will discuss the complex manifold structure on $\mathcal{H}_{\text {inv }}$, therefore, on $\mathcal{F}_{\text {inv }}$. The complex manifold structure which we will discuss is the integration of the almost complex structure $\mathcal{J}$ which we discussed in the previous section.

Consider

$$
\mathcal{H}=\{h \mid h \text { is a quasisymmetric circle homeomorphism with } h(1)=1\} .
$$

Since $\mathcal{H}_{\text {inv }}$ is the subspace of all $h \in \mathcal{H}$ satisfying the linear equation (11), it is a smooth submanifold of $\mathcal{H}$. The almost complex structure $\mathcal{J}: \mathcal{H}_{\text {inv }} \rightarrow \mathcal{H}_{\text {inv }}$ is the restriction of the almost complex structure $\mathcal{J}: \mathcal{H} \rightarrow \mathcal{H}$. Thus, to have our original 
goal, we can discuss the complex manifold structure on $\mathcal{H}$ which is the integration of the almost complex structure $\mathcal{J}: \mathcal{H} \rightarrow \mathcal{H}$.

In the following, we will discuss the Teichmüller structure on $\mathcal{H}$ and its embedding to the space of all holomorphic functions on the unit disk. This will give a complex manifold structure on $\mathcal{H}$ (as well as $\mathcal{F}$ ). Furthermore, we will prove that the almost complex structure induced from this complex manifold structure is the Hilbert transform $\mathcal{J}$. Thus, the induced complex manifold structure on $\mathcal{H}_{\text {inv }}$ (as well as on $\mathcal{F}_{\text {inv }}$ ) is the integral of the almost complex structure on $\mathcal{H}_{\text {inv }}$ (as well as $\mathcal{F}_{\text {inv }}$ ) defined by the Hilbert transform $\mathcal{J}$.

The covering map from the real line $\mathbf{R}$ to the unit circle $T$ is

$$
\pi(x)=e^{2 \pi i x}: \mathbf{R} \rightarrow T .
$$

So any map in $\mathcal{H}$ can be lifted to a periodic quasisymmetric homeomorphism $H$ of R. We denote the space of all lifting map to be $\widetilde{\mathcal{H}}$, i.e.,

$$
\begin{gathered}
\widetilde{\mathcal{H}}=\{H \mid H \text { is a quasisymmetric homeomorphism of } \mathbf{R} \\
\text { with } H(x+1)=H(x)+1 \text { and } H(0)=0\} .
\end{gathered}
$$

Since $h$ and $H$ are quasisymmetric, we can extend $h$ and $H$ to quasiconformal homeomorphisms of $\overline{\mathbf{C}}$. Let $f(z)$ be the Beurling-Ahlfors extension of $H$ (see [1]), then $f(z+1)=f(z)+1$ and the corresponding $\mu=f_{\bar{z}} / f_{z}$ satisfies the condition $\mu(z)=\mu(z+1)$. The converse of the previous argument is also true. Suppose $\mu(z)$ is a measurable function defined on $\overline{\mathbf{C}}$ with $\|\mu\|_{\infty}<1$. Such a function is called a Beltrami coefficient. Consider the Beltrami equation

$$
f_{\bar{z}}=\mu f_{z} .
$$

A solution of the Beltrami equation (14) is called normalized if it fixes 0,1 , and $\infty$. The normalized solution is unique for any given Beltrami coefficient $\mu$.

Lemma 1. Suppose $\mu$ is a Beltrami coefficient satisfying that $\mu(z+1)=\mu(z)$ for all $z \in \mathbf{C}$. Suppose $f(z)$ is the normalized solution of the corresponding Beltrami equation (14). Then

$$
f(z+1)=f(z)+1, \quad \forall z \in \mathbf{C} .
$$

Proof. The following function $\mathcal{P}$ and $\mathcal{T}$ are two operators defined in [1].

$$
P \mu(\zeta)=-\frac{1}{\pi} \iint \mu(z)\left(\frac{1}{z-\zeta}-\frac{1}{z}\right) d x d y
$$

and

$$
T \mu(\zeta)=\lim _{\epsilon \rightarrow 0}-\frac{1}{\pi} \iint_{|z-\zeta|>\epsilon} \frac{\mu(z)}{(z-\zeta)^{2}} d x d y
$$

Since $\mu(z+1)=\mu(z), \mathcal{P}(\mu(z))=\mathcal{P}(\mu(z+1))$ and $\mathcal{T}(\mu(z))=\mathcal{T}(\mu(z+1))$. We have that both $f(z)$ and $f(z+1)-1$ are the normalized solution of the corresponding Beltrami equation (14), so $f(z+1)=f(z)+1$. We proved the lemma.

Consider the space $\mathcal{M}_{r}$ of all measurable functions $\mu$ defined on the Riemann sphere $\overline{\mathbf{C}}$ satisfying

- $\mu(z+1)=\mu(z)$;

- $\mu(\bar{z})=\overline{\mu(z)}$ for all $z \in \mathbf{C}$;

- $\|\mu\|_{\infty}<1$. 
For any $\mu \in \mathcal{M}_{r}$, the restriction of the normalized solution $f$ of the corresponding Betrami equation (14) to the real line $\mathbf{R}$ is in $\tilde{\mathcal{H}}$. For any $H \in \tilde{\mathcal{H}}$, let $f(z)$ be the Beurling-Ahlfors extension of $H$ (see [1]), then $f(z+1)=f(z)+1$ and the corresponding $\mu=f_{\bar{z}} / f_{z}$ is in $\mathcal{M}_{r}$. Thus every map in $\tilde{\mathcal{H}}$ can be thought as the restriction of the normalized solution $f$ of a Betrami equation (14) for some Beltrami coefficient $\mu \in \mathcal{M}_{r}$.

Consider the space $\mathcal{M}_{c}$ of all measurable functions $\mu$ defined on the Riemann sphere $\overline{\mathbf{C}}$ satisfying

- $\mu(z)=\frac{z^{2}}{\bar{z}^{2}} \overline{\mu(1 / \bar{z})}$ for any $z \in \mathbf{C}$;

- $\|\mu\|_{\infty}<1$.

For any $\mu \in \mathcal{M}_{c}$, the restriction of the normalized solution $g$ of the corresponding Betrami equation (14) to the unit circle $T$ is in $\mathcal{H}$. Similarly, every map in $\mathcal{H}$ can be thought as the restriction of the normalized solution $g$ of a Betrami equation (14) for some Beltrami coefficient $\mu \in \mathcal{M}_{c}$.

For any $\mu \in \mathcal{M}_{r}$, we use $f_{\mu}$ to denote the normalized solution of the corresponding Beltrami equation (14). Then $f_{\mu}$ maps the real line $\mathbf{R}$ onto itself. For any $\mu \in \mathcal{M}_{c}$, we use $g_{\mu}$ to denote the normalized solution of the Beltrami equation (14). Then $g_{\mu}$ maps the unit circle $T$ onto itself.

Definition 9. (Teichmüller Equivalence) Suppose $\mu$ and $\nu$ in $\mathcal{M}_{r}$. We say that they are equivalent, denoted as $\mu \sim_{r} \nu$ if $f_{\mu}(x)=f_{\nu}(x)$ for all $x \in \mathbf{R}$. We use $[\mu]_{r}$ to denote the $\sim_{r}$-equivalent class of $\mu \in \mathcal{M}_{r}$. We define the Teichmüller space

$$
\mathcal{T}_{r}=\left\{[\mu]_{r} \mid \mu \in \mathcal{M}_{r}\right\}
$$

as the space of all $\sim_{r}$-equivalence classes. Suppose $\mu$ and $\nu$ in $\mathcal{M}_{c}$. We say that they are equivalent, denoted as $\mu \sim_{c} \nu$ if $g_{\mu}(z)=g_{\nu}(z)$ for all $z \in T$. We use $[\mu]_{c}$ to denote the $\sim_{c}$-equivalent class of $\mu \in \mathcal{M}_{c}$. We define the Teichmüller space

$$
\mathcal{T}_{c}=\left\{[\mu]_{c} \mid \mu \in \mathcal{M}_{c}\right\}
$$

as the space of all $\sim_{c^{-}}$equivalence classes.

From the definition and the standard theory for quasiconformal mappings (see [1]), there is a one-to-one and onto correspondence between $\tilde{\mathcal{H}}$ and $\mathcal{T}_{r}$. Therefore, $\tilde{\mathcal{H}}$ can be thought as another representation of the Teichmüller space $\mathcal{T}_{r}$. There is a oneto-one and onto correspondence between $\mathcal{H}$ and $\mathcal{T}_{c}$. Therefore, $\mathcal{H}$ can be thought as another representation of the Teichmüller space $\mathcal{T}_{c}$. We also know that there is a oneto-one and onto correspondence between $\tilde{\mathcal{H}}$ and $\mathcal{H}$. Therefore, there is a one-to-one and onto correspondence

$$
\tau: \mathcal{T}_{c} \rightarrow \mathcal{T}_{r}
$$

9.1. Complex structures on $\mathcal{T}_{c}, \mathcal{T}_{r}$ and $\mathcal{T}\left(\Delta^{*}\right)$. The space $\mathcal{T}_{r}$ is a subspace of the universal Teichmüller space. From Bers' embedding (refer to [7]), there is a natural complex manifold structure on $\mathcal{T}_{r}$ which is given by considering the Schwarzian derivative

$$
S\left(f^{\mu}\right)=\left(\frac{\left(f^{\mu}\right)^{\prime \prime}}{\left.f^{\mu}\right)^{\prime}}\right)^{\prime}-\frac{1}{2}\left(\frac{\left(f^{\mu}\right)^{\prime \prime}}{\left(f^{\mu}\right)^{\prime}}\right)^{2}
$$

on the lower-half plane $H^{*}$, where $f^{\mu}$ is the normalized solution of the Beltrami equation (14) with the Beltrami coefficient $\tilde{\mu}=\mu$ on the upper-half plane and $\tilde{\mu}=0$ on the lower-half plane. 
Let $\Delta=\{z \in \mathbf{C}|| z \mid<1\}$ be the open unit disk and $\Delta^{*}=\Delta \backslash\{0\}$ be the punctured disk. Let $T\left(\Delta^{*}\right)$ be the Teichmüller space of Riemann surfaces with the basepoint $\Delta^{*}$ i.e.,

$$
\mathcal{T}\left(\Delta^{*}\right)=\left\{[\mu]_{\Delta^{*}} \mid \mu \in \mathcal{M}\right\}
$$

where $\mathcal{M}$ is the unit ball of $L_{\infty}\left(\Delta^{*}\right)$, is the space of all Teichmüller equivalence classes $[\mu]_{\Delta^{*}}$. Here $\mu$ and $\nu$ are said to be Teichmüller equivalent, denoted as $\mu \sim_{\Delta^{*}} \nu$, if two unique solutions $f$ and $g$ of the Beltrami equation (14) with the Beltrami coefficients $\mu$ and $\nu$ in $\Delta$ and $1 / \overline{\mu(1 / \bar{z})}$ and $1 / \overline{\nu(1 / \bar{z})}$ in $\overline{\mathbf{C}} \backslash \Delta$ fixing $-1,1, i$ agree on the unit circle $T$ and on 0 , that is, $f|T=g| T$ and $f(0)=g(0)$.

On $\mathcal{T}_{c}$ we can define the Teichmüller metric by

$$
d_{\mathcal{T}}(x, y)=\frac{1}{2} \inf _{\mu \in x, \nu \in y} \log K\left(f_{\mu} \circ f_{\nu}^{-1}\right),
$$

where $f_{\mu}$ and $f_{\nu}$ are normalized solutions of Beltrami equation (14) with beltrami coefficients $\mu$ and $\nu$ and $K\left(f_{\mu} \circ f_{\nu}^{-1}\right)$ is the quasiconformal dilatation of $f_{\mu} \circ f_{\nu}^{-1}$.

Let $d^{*}(\cdot, \cdot)$ be the Teichmüller distance on $T\left(\Delta^{*}\right)$.

Theorem 10. The space $\left(\mathcal{T}_{c}, d_{\mathcal{T}}\right)$ is isometric to the space $\left(\mathcal{T}\left(\Delta^{*}\right), d^{*}\right)$.

Proof. For any $x=[\mu]_{c} \in \mathcal{T}_{c}, h=f_{\mu} \mid T$ is in $\mathcal{H}$. The map $h$ fixes 1 . There exists a Möbius map

$$
M_{a}(z)=\frac{z+a}{1+\bar{a} z} \cdot \frac{1+\bar{a}}{1+a}
$$

such that $M_{a} \circ h$ is a homeomorphism of $T$ fixing $1, i$ and -1 . Thus $M_{a} \circ h$ represents the equivalent class of the Beltrami coefficient of $M_{a} \circ f_{\mu}$ in $\mathcal{T}\left(\Delta^{*}\right)$. Moreover, $M_{a}^{-1} \circ h$ will map a point $h \in T\left(\Delta^{*}\right)$ to a point in $\mathcal{T}_{c}$. This correspondence preserves the metrics $d_{\mathcal{T}}$ and $d^{*}$.

The pull back of the complex structure, given by the Schwarzian derivative, on $\mathcal{T}_{r}$ by $\tau$ gives a complex complex structure on $\mathcal{T}_{c}$. From Theorem 10 , this also gives a complex structure on $\mathcal{T}\left(\Delta^{*}\right)$. Therefore, we have a complex manifold structure on $\mathcal{H}$.

9.2. Almost complex structure. The tangent vector $V$ in the tangent space of $\mathcal{T}_{r}$ at the identity has the form

$$
V(x)=\frac{-1}{\pi} \iint_{\mathbf{C}} \frac{\mu(\zeta)}{\zeta(\zeta-1)(\zeta-x)} d \xi d \eta
$$

for any real number $x$ and for some $\mu$ which is symmetric, periodic and in $L_{\infty}(H) /$ $N(H)$ (see [7]). So multiplication by $-i$ on Beltrami coefficients $\mu$ determines the standard almost complex structure on Teichmuller space $\mathcal{T}_{r}$. From the calculation in Section 7, the Hilbert transform gives the same almost complex structure on $\mathcal{V}$. This observation for the universal Teichmüller space is due to Kerckhoff (see, for example, [23]).

So the pull back of Hilbert transform by $\tau: \mathcal{T}_{c} \rightarrow \mathcal{T}_{r}$ gives an almost complex structure on the tangent space of $\mathcal{T}_{c}$ at the identity. From Theorem 7 in Section 7 , the Hilbert transform keeps the condition (9) and (10) which implies the pull back of Hilbert transform by $\tau$ gives an almost complex structure on the tangent space $\mathcal{F}_{\text {inv }}$ at identity, whose integration is the complex manifold we just discussed in Section 8. 


\section{The Teichmüller metric and Kobayashi's metric}

Since $\mathcal{T}_{c}$ is a complex manifold, we can define Kobayashi's metric on $\mathcal{T}_{c}$ (see [6]). Since the Teichmüller metric is equal to Kobayashi's metric on $\mathcal{T}\left(\Delta^{*}\right)$ (see [6]), from Theorem 8, the Teichmüller metric is equal to Kobayashi's metric on $\mathcal{T}_{c}$.

The restriction of a Möbius transformation

$$
M_{a}(z)=\frac{z+a}{1+\bar{a} z} \cdot \frac{1+\bar{a}}{1+a}
$$

to the unit circle $T$ maps $T$ onto $T$ and fixes 1 . It is the boundary map of a quasiconformal map $f_{\mu}$ of $\overline{\mathbf{C}}$ fixing 0,1 , and $\infty$ for a $[\mu] \in \mathcal{T}_{c}$. Note that $\mu$ is not $\sim_{c}$-equivalent to $\mu_{0} \equiv 0$. But $\mu$ is the Teichmüller equivalent to $\mu_{0} \equiv 0$ in the universal Teichmüller space $\mathcal{T}(\Delta)$.

Let $\mathcal{P}: \mathcal{T}\left(\Delta^{*}\right) \rightarrow \mathcal{T}(\Delta)$ be the forgetful map since $\Delta^{*} \subset \Delta$. It is a holomorphic split submersion, that means that for every point $x \in \mathcal{T}(\Delta)$, there is a neighborhood $U$ about $x$ and a holomorphic map $s: U \rightarrow \mathcal{T}\left(\Delta^{*}\right)$ such that $\mathcal{P} \circ s=$ id.

Suppose $[\mathrm{id}]=[0]_{\Delta}$ is the basepoint of $\mathcal{T}(\Delta)$. Let $\mathcal{K}=\mathcal{P}^{-1}([\mathrm{id}])$. Then

$$
\mathcal{K}=\left\{M_{a}(z) \mid a \in \Delta\right\}
$$

is a one dimensional complex manifold of $\mathcal{T}\left(\Delta^{*}\right)$ conformally equivalent to the hyperbolic disk $\Delta$. Therefore, we have Kobayashi's metric on $\mathcal{K}$ and the restriction of Teichmüller metric on $\mathcal{K}$, which we denote as $d^{*}$. Comparing these two metrics is an interesting problem. Gardiner and Lakic [8, 9] have studied this problem infinitesimally in a more general setting as follows:

Definition 10. Suppose $\Omega$ is a domain contained in $\overline{\mathbf{C}}$ with three or more boundary points. Its Poincare density is denoted as

$$
\rho_{\Omega}(p)=\frac{\rho_{\Delta}(z)}{\left|\pi^{\prime}(z)\right|}
$$

where $\rho_{\Delta}(z)=\frac{|d z|}{1-|z|^{2}}$ and $\pi: \Delta \rightarrow \Omega$ is the universal covering and $p=\pi(z)$.

Definition 11. The Teichmüller density $\lambda_{\Omega}(p)$ evaluated at a point $p$ in $\Omega$ is

$$
\lambda_{\Omega}(p)=\inf \left\{\|\bar{\partial}(\widetilde{V})\|_{\infty}\right\},
$$

where the infimum is taken over all continuous vector fields $\widetilde{V}(z) \frac{\partial}{\partial z}$ for which $\widetilde{V}(p)=1$ and $\widetilde{V}(z)=0$ for all $z$ on the boundary of $\Omega$.

Suppose $\mathcal{P}: \mathcal{T}(\Omega-p) \rightarrow \mathcal{T}(\Omega)$ is the forgetful map, which is a holomorphic split submersion, and suppose [id] is the basepoint of $\mathcal{T}(\Omega)$. Consider the fiber $\mathcal{K}=\mathcal{P}^{-1}([\mathrm{id}])$. In [2], Bers shows that when $\Omega$ is of finite analytic type, $\mathcal{K}$ is conformally equivalent to the universal covering of $\Omega$. In [8], Gardiner and Lakic showed that the infinitesimal Kobayashi's metric at the basepoint for $\mathcal{K}$ is equal to the Poincaré density $\rho_{\Omega}(p)$ at $p$ in $\Omega$. The infinitesimal Teichmüller's metric at the basepoint for $\mathcal{K}$ is the Teichmüller density $\lambda_{\Omega}(p)$. Furthermore, they have the following comparison.

Theorem 11. (Gardiner and Lakic [8]) The Teichmüller density $\lambda_{\Omega}(p)$ and the Kobayashi's density $\rho_{\Omega}(p)$ are equivalent. In particular,

$$
\frac{1}{2} \rho_{\Omega}(p) \leq \lambda_{\Omega}(p) \leq \rho_{\Omega}(p)
$$


The right hand side of the inequality is an easy corollary of Slodkowski's extension theorem [21] in the holomorphic motion theory (see also [6]). And they use the Poincare theta series of a quadratic differential to show the left hand side. The reader who is interested in this theorem can refer to [8] for more details.

For $\Omega=\Delta$ and $p=0$, Gardiner and Lakic have a result in [9] recently as follows. We give a proof of this result in this section.

Theorem 12. (Gardiner and Lakic [9]) Then

$$
\lambda_{\Delta}(0)=\frac{1}{2} \rho_{\Delta}(0)=\frac{1}{2} .
$$

Proof. Since $\frac{1}{2} \rho_{\Delta}(0)=\frac{1}{2}$, from Theorem 9 , we have that

$$
\frac{1}{2} \rho_{\Delta}(0) \leq \lambda_{\Delta}(0)
$$

So we only need to show that $\lambda_{\Delta}(0) \leq \frac{1}{2}$ as follows.

Since the fiber $\mathcal{K}$ is the hyperbolic disk $\Delta$ conformally, we consider the holomorphic motion

$$
h(t, z)=\left\{\begin{array}{ll}
z, & |z| \geq 1 \\
z+t(1-|z|), & |z| \leq 1
\end{array}: \Delta \times \mathcal{C} \rightarrow \overline{\mathcal{C}} .\right.
$$

The tangent vector of this motion on $|z| \leq 1$ is

$$
V(z)=\left.\frac{d(h(t, z))}{d t}\right|_{t=0}=1-|z|=1-z^{\frac{1}{2}} \bar{z}^{\frac{1}{2}} .
$$

So $V(0)=1$ and $V(z)=0$ for $|z|=1$.

For any $z \in \Delta$,

$$
|\bar{\partial} V(z)|=\left|\frac{1}{2} \frac{z^{\frac{1}{2}}}{z^{\frac{1}{2}}}\right|=\frac{1}{2} .
$$

Therefore, $\|\bar{\partial} V\|_{\infty}=\frac{1}{2}$ and

$$
\lambda_{\Delta}(0)=\inf \left\{\|\bar{\partial}(\widetilde{V})\|_{\infty}\right\} \leq \frac{1}{2},
$$

where the infimum is taken over all continuous vector fields $\widetilde{V}(z) \frac{\partial}{\partial z}$ for which $\widetilde{V}(0)=1$ and $\widetilde{V}(z)=0$ for all $z$ on the boundary of $\Delta$.

The above theorems are about the estimation of the Kobayashi's density and the Teichmüller density on the fiber $\mathcal{K}$ at the basepoint for the fiber $\mathcal{K}$. In this section, we prove that this kind of estimations will not exist in the global meaning. That is, we will prove that from the global point of view, Theorems 9 and 10 will not hold on the fiber $\mathcal{K}$. More precisely, we prove that

Theorem 13. On the fiber $\mathcal{K}$, the global Teichmüller metric $d_{T e i}$ and the global Kobayashi's metric $d_{K o b}$ on $\mathcal{K}$ are not equivalent.

Proof. We use the upper-half plane model for the universal Teichmüller space $\mathcal{T}(H)$. Then $p=i$. Denote $H^{*}=H-\{i\}$. Then we have the forgetful map $\mathcal{P}: \mathcal{T}\left(H^{*}\right) \rightarrow \mathcal{T}(H)$. Let [id] be the basepoint for $\mathcal{T}(H)$. Then the fiber

$$
\mathcal{K}=\mathcal{P}^{-1}([\mathrm{id}])=\left\{f_{w}(z) \mid f(x)=x, \forall x \in R, f(i)=w\right\}=\left\{M_{w}(z)=\eta z+\xi\right\}=H,
$$

where $w=\xi+\eta i \in H$. Note that $M_{w}(i)=w$ and $M_{w}(\infty)=\infty$. 
Given any number $K>1$. Suppose $f_{K}$ is a quasi-conformal self homeomorphism of $H$ such that $f_{K}(x)=x$ for any real $x \in \mathbf{R}$ and $f_{K}(i)=K i$. Take the Beltrami coefficient $\mu_{f_{K}}(z)=\bar{\partial} f_{K}(z) / \partial f_{K}(z)$. Let $\tau_{K}=\left[\mu_{f_{K}}\right]_{H^{*}}$ be a point in $\mathcal{T}\left(H^{*}\right)$. That is, $\tau_{K}$ is the equivalence class of all Beltrami coefficient $\nu$ on $H$ such that $f_{\nu} \mid \mathbf{R}=$ id and $f_{\nu}(i)=K i$. Clearly, $\tau_{K}$ is a point in the fiber $\mathcal{K}$. Let $[\mathrm{id}]_{H^{*}}$ be the basepoint of $\mathcal{T}\left(H^{*}\right)$. The Kobayashi's distance $d_{\mathrm{Kob}}\left([\mathrm{id}]_{H^{*}}, \tau_{K}\right)$ on the fiber $\mathcal{K}$ is equal to the Poincaré distance $d_{\mathrm{Hyp}}(i, K i)=\log K$ on the hyperbolic upper-half plane $H$.

We will use Strebel's extremal example to get a upper bound of the Teichmüller distance $d_{\mathrm{Tei}}\left([\mathrm{id}]_{H^{*}}, \tau_{K}\right)$ on the fiber $\mathcal{K}$. Let

$$
z=\pi(w)=\frac{2}{\pi} \log r+4 i \theta, \quad w=r e^{2 \pi i \theta}, r>0,0 \leq \theta \leq \frac{1}{2} .
$$

Then it maps the upper-half plane $H$ to the horizontal strip

$$
A=\{z=x+y i \mid-\infty<x<\infty, 0 \leq y \leq 2\} .
$$

It maps the positive real axis to the real line; the positive imaginary axis to the line $y=1$; the negative real line to the line $y=2$.

Let $g: A \rightarrow A$ be a self quasi-conformal homeomorphism of $A$ defined as

$$
g(z)= \begin{cases}z+\frac{2}{\pi} \frac{z-\bar{z}}{2 i} \log K, & 0 \leq y \leq 1 \\ z+\frac{2}{\pi} \log K\left(2-\frac{z-\bar{z}}{2 i}\right), & 1 \leq y \leq 2\end{cases}
$$

Then the Beltrami coefficient is

$$
\mu_{g}=\frac{-\frac{1}{\pi i} \log K}{1+\frac{1}{\pi i} \log K} .
$$

Thus the quasiconformal dilatation

$$
K(g)=\left(\sqrt{1+\left(\frac{1}{\pi} \log K\right)^{2}}+\frac{1}{\pi} \log K\right)^{2} .
$$

Define $f=\pi^{-1} \circ g \circ \pi$. It is a quasiconformal self homeomorphism of the upperhalf plane $H$. We have that $K(f)=K(g)$. Since $f \mid \mathbf{R}=\mathrm{id}$ and $f(i)=K i$, the Beltrami coefficient $\mu_{f} \in \tau_{K}$. This gives us that the Teichmüller distance

$$
d_{\mathrm{Tei}}\left([\mathrm{id}]_{H^{*}}, \tau_{K}\right) \leq \log K(f)=2 \log \left(\sqrt{1+\left(\frac{1}{\pi} \log K\right)^{2}}+\frac{1}{\pi} \log K\right) .
$$

Hence

$$
d_{\mathrm{Tei}}\left([\mathrm{id}]_{H^{*}}, \tau_{K}\right) \approx 2 \log \left(\frac{2}{\pi} \log K\right), \quad \text { as } K \rightarrow \infty .
$$

Thus we have that

$$
\frac{d_{\mathrm{Tei}}\left([\mathrm{id}]_{H^{*}}, \tau_{K}\right)}{d_{\mathrm{Kob}}\left([\mathrm{id}]_{H^{*}}, \tau_{K}\right)} \approx \frac{2 \log \left(\frac{2}{\pi} \log K\right)}{\log K} \rightarrow 0, \quad \text { as } K \rightarrow \infty .
$$

We proved our theorem. 


\section{References}

[1] Ahlfors, L. V.: Lectures on quasiconformal mappings. - Univ. Lecture Ser. 38, 2nd edition, Amer. Math. Soc., Providence, RI, 2006.

[2] Bers, L.: Fiber spaces over Teichmüller spaces. - Acta Math. 130, 1973, 89-126.

[3] Earle, C. J.: Teichmüller spaces as complex manifolds: Warwick, 1992. - In: Teichmüller theory and moduli problem, Ramanujan Math. Soc. Lect. Notes Ser. 10, 2010, 5-33.

[4] Gardiner, F. P., and W. J. HarveY: Universal Teichmüller space. - In: Handbook of complex analysis: Geometric function theory, Vol. 1, North-Holland, Amsterdam, 2002, 457-492.

[5] Gardiner, F. P., and Y. JiAng: Asymptotically affine and asymptotically conformal circle endomorphisms. - In: Infinite dimensional Teichmüller spaces and moduli spaces, edited by Ege Fujikawa, RIMS Kôkyûroku Bessatsu B17, 2010, 37-53.

[6] Gardiner, F. P., Y. JiAng, and Z. WANG: Holomorphic motions and related topics. - In: Geometry of Riemann surfaces, London Math. Soc. Lecture Note Ser. 368, 2010, 156-193.

[7] Gardiner, F. P., and N. Lakic: Quasiconformal Teichmüller theory. - Amer. Math. Soc., Providence, Rhode Island, 2000.

[8] Gardiner, F. P., and N. Lakic: Comparing Poincare desities. - Ann. of Math. (2) 154, 2001, $245-267$.

[9] Gardiner, F. P., and N. LakiC: Tracking a moving point on a Riemann surface. - Trans. Amer. Math. Soc. (to appear).

[10] Gardiner, F. P., and D. P. Sullivan: Symmetric structures on a closed curve. - Amer. J. Math. 114, 1992, 683-736.

[11] JiAng, Y.: Generalized Ulam-von Neumann transformations. - Ph.D. Thesis, Graduate School of CUNY and UMI publication, 1990.

[12] JiAnG, Y.: Geometry of geometrically finite one-dimensional maps. - Comm. Math. Phys. 156:3, 1993, 639-647.

[13] JiAnG, Y.: Renormalization and geometry in one-dimensional and complex dynamics. - Adv. Ser. Nonlinear Dynam. 10, World Sci. Publ., River Edge, NJ, 1996.

[14] JiAng, Y.: Smooth classification of geometrically finite one-dimensional maps. - Trans. Amer. Math. Soc. 348:6, 1996, 2391-2412.

[15] Jiang, Y.: On rigidity of one-dimensional maps. - Contemp. Math. 211, 1997, 319-431.

[16] JIANG, Y.: A proof of existence and simplicity of a maximal eigenvalue for Ruelle-PerronFrobenius operators. - Lett. Math. Phys. 48:3, 1999, 211-219.

[17] JIANG, Y.: Lecture notes in dynamical systems and quasiconformal mappings. - A course given in Department of Mathematics at CUNY Graduate Center, spring semester, 2009.

[18] JiAng, Y.: Symmetric invariant measures. - Contemp. Math. 575, 2012, 211-218.

[19] JIANG, Y.: Teichmüller structures and dual geometric Gibbs type measure theory for continuous potentials. - arXiv0804.3104v3.

[20] NAG, S.: On the tangent space to the universal Teichmüller space. - Ann. Acad. Sci. Fenn. Ser. A I Math. 18:2, 1993, 377-393.

[21] Slodkowski, S.: Holomorphic motions and polynomial hulls. - Proc. Amer. Math. Soc. 111:2, 1991, 347-355.

[22] Varadhan, S. R. S.: Probability theory. - Courant Lect. Notes Math. 7, Amer. Math. Soc., Providence, RI, 2001.

[23] Wolpert, S. A.: Thurston's Riemannian metric for Teichmüller space. - J. Differential Geom. $23,1986,143-174$.

Received 11 January $2012 \bullet$ Accepted 3 September 2012 